Review began 09/04/2021 Review ended 09/11/2021 Published 09/20/2021

๑) Copyright 2021

Umeh et al. This is an open access article distributed under the terms of the Creative Commons Attribution License CC-BY 4.0. which permits unrestricted use, distribution, and reproduction in any medium, provided the original author and source are credited.

\section{Predictors of Mortality in COVID-19 Patients in Southern California - Retrospective Multicenter Study}

Chukwuemeka Umeh $^{1}$, Stella Maguwudze ${ }^{1}$, Adrian Torbela ${ }^{1}$, Shipra Saigal ${ }^{1}$, Harpreet Kaur ${ }^{1}$, Shadi Kazourra ${ }^{1}$, Mahendra Aseri ${ }^{2}$, Rakesh Gupta ${ }^{3}$, Sumanta Chaudhuri ${ }^{1}$, Rahul Gupta ${ }^{1}$

1. Internal Medicine, Hemet Global Medical Center, Hemet, USA 2. Data Engineering \& Business Intelligence, Hemet Global Medical Center, Hemet, USA 3. Pulmonary and Critical Care Medicine, Hemet Global Medical Center, Hemet, USA

Corresponding author: Chukwuemeka Umeh, emmyumeh@yahoo.com

\section{Abstract \\ Introduction}

The majority of patients infected with coronavirus disease 2019 (COVID-19) recover from the illness after suffering mild to moderate symptoms, while approximately $20 \%$ progress to severe or critical disease, which may result in death. Understanding the predictors of severe disease and mortality in COVID-19 patients will help to risk stratify patients and improve clinical decision making. US data to inform this understanding are, however, scarce. We studied predictors of COVID-19 mortality in a cohort of 1,116 hospitalized patients in Southern California in the United States.

\section{Methods}

We conducted a retrospective cohort study of COVID-19 patients admitted at two hospitals in Southern California United States between March 2020 and March 2021. Bivariate and multivariate analyses of the relationship between mortality and other variables such as demographics, comorbidities, and laboratory values were performed, with a p-value of 0.05 considered as significant.

\section{Results}

The analysis involved 1,116 COVID-19 patients, of which $51.5 \%$ were males and $48.5 \%$ were females. Of the 1,116 patients, $81.6 \%$ were whites, $7.2 \%$ were blacks, and $11.2 \%$ were other races. After adjusting for covariables, age $(\mathrm{p}<0.001)$, admission to intensive care unit $(\mathrm{p}<0.001)$, use of remdesivir $(\mathrm{p}=0.018)$, C-reactive protein $(C R P)$ levels $(p<0.001)$, and lactate dehydrogenase $(\mathrm{LDH})$ levels $(\mathrm{p}=0.039)$ were independently associated with mortality in our study. Gender, race, body mass index, presence of co-morbidities such as diabetes and hypertension, and use of steroid, statin, calcium channel blockers, angiotensin-converting enzyme inhibitors or angiotensin receptor blockers were not associated with mortality in the multivariate analysis.

\section{Conclusion}

In the cohort we studied, admission to intensive care unit was associated with decreased mortality while older age, use of remdesivir, and high levels of CRP and LDH were associated with increased mortality in COVID-19 patients.

Categories: Internal Medicine, Infectious Disease, Pulmonology

Keywords: intensive care unit (icu), creatine phosphokinase (cpk), lactate dehydrogenase (ldh), california, remdesivir, mortality, covid-19

\section{Introduction}

Coronavirus disease 2019 (COVID-19) is caused by a novel coronavirus belonging to the family Coronaviridae and has caused significant morbidity and mortality globally [1]. The first cases were reported in China in December 2019 and a pandemic was declared on March 11, 2020, by the World Health Organization [1]. The infection mainly presents as fever, body aches, shortness of breath, malaise, and dry cough; some patients present with gastrointestinal symptoms such as diarrhea [1]. Most people infected with COVID-19 virus have mild disease and recover, but about $20 \%$ of patients go on to develop severe or critical disease that may result in death [2]. COVID-19 was first reported in the United States on January 21, 2020 [3]. Since then, there have been more than 32 million confirmed cases and over 500,000 COVID-19 deaths in the United States as of May 22, 2021. Greater than 10\% of these deaths occurred in California [4].

Although prior COVID-19 studies on predictors of severe disease and mortality have been published from different locations globally, there is paucity of data from the United States. We now know from prior studies 
that advanced age, elevated inflammatory markers, presence of comorbidities such as diabetes and hypertension, and presence of ground glass opacities on imaging are associated with increased risk of mortality [5-8]. However, we do not clearly understand to what extent these factors predict death or survival in patient with COVID-19 in California considering the paucity of studies from the United States. Understanding the predictors of severe disease or mortality in COVID-19 patients will help to risk stratify patients and inform clinical decisions. To our knowledge, this is the first study in Southern California, which examines predictors of mortality in patients hospitalized with COVID-19.

\section{Materials And Methods}

This is a retrospective study of 1,116 COVID-19 patients who were admitted in two hospitals in Southern California in the United States between March 2020 and March 2021. The study includes all patients who sought care at the two facilities for COVID-19 and non-COVID-19-related symptoms and were diagnosed with COVID-19 through a positive polymerase chain reaction nasopharyngeal swab. For COVID-19 patients who were admitted in the hospital more than once during the study period for COVID-19 symptoms, their last hospital admission data were used for the study. We used COVID-19 patients' last hospital admission for COVID-19-related symptoms because some patients died from COVID-19-related complications during their subsequent hospital admission. Using the last hospital admission prevented a misclassification bias because we would otherwise have misclassified some patients who died as having survived if we only used their initial hospitalization for COVID-19 symptoms. Relevant deidentified data were extracted from the electronic medical record including patients age, sex, race, ethnicity, marital status, comorbidities, laboratory results on admission, date of admission, date of discharge, medications they received while on admission, and disposition at discharge. The decision on each patient's level of care and what treatment to start on each patient was done by the hospitals' COVID-19 team consisting of a pulmonary and critical care specialist and hospitalists, in consultation with an infectious disease specialist and pharmacist. Every COVID-19 patient was seen by one of the members of the COVID-19 team.

Univariate analysis of study variables was done using means and percentages. Bivariate analysis of the relationship of different study variables with mortality was done using chi-square and t-test, with a p-value of 0.05 considered as significant. Finally, backward selection cox regression analysis was done using mortality as a dependent variable. All other variables such as patients' age, sex, race, ethnicity, marital status, comorbidities, medication that patients received while in the hospital, and laboratory results on admission were included as independent variables. The effect was expressed in terms of hazards ratio with 95\% confidence interval. Statistical analysis was done using IBM SPSS version 27 (IBM Corp, Armonk, NY). The study was approved by WIRB-Copernicus Group institutional review board (IRB) and the study IRB approval number is 13410516.

\section{Results}

\section{Baseline study characteristics}

The analysis involved 1,116 COVID-19 patients, of which $51.5 \%$ were males and $48.5 \%$ females. Of the 1,116 patients, $81.6 \%$ were whites, $7.2 \%$ were blacks, and $11.2 \%$ were other races. Nineteen percent of the patients were placed on a ventilator during their hospital admission and $26.4 \%$ of the patients died during hospital admission.

\section{Bivariate analysis}

In the bivariate analysis of categorical variables, male sex $(\mathrm{p}<0.001)$, ventilator dependence $(\mathrm{p}<0.001)$, admission to intensive care unit (ICU) ( $p<0.001$ ), presence of hypertension ( $p=0.009)$, chronic kidney disease $(\mathrm{p}<0.001)$, acute kidney injury $(\mathrm{p}<0.001)$, congestive heart failure $(\mathrm{p}<0.001)$, chronic obstructive pulmonary disease $(\mathrm{p}=0.005)$, coronary artery disease $(\mathrm{p}<0.001)$, beta blockers use $(\mathrm{p}=0.015)$, calcium channel blocker use $(\mathrm{p}=0.01)$, and steroid use $(\mathrm{p}<0.001)$ were associated with increased mortality (Table 1$)$. Patients' race, diabetes, cerebrovascular accident, and use of angiotensin-converting-enzyme inhibitors (ACEi) or angiotensin receptor blockers (ARBs) were not associated with increased mortality (Table 1).

\begin{tabular}{|c|c|c|c|}
\hline Variable & Expired & Survived & p-Value \\
\hline \multicolumn{4}{|l|}{ Gender } \\
\hline Male & $180(31.3 \%)$ & $395(68.7 \%)$ & $<0.001$ \\
\hline Female & $115(21.3 \%)$ & $426(78.7 \%)$ & \\
\hline \multicolumn{4}{|l|}{ Race } \\
\hline White & $239(26.2 \%)$ & $672(73.8 \%)$ & 0.156 \\
\hline Black & $16(20 \%)$ & $64(80 \%)$ & \\
\hline Others & $40(32 \%)$ & $85(68 \%)$ & \\
\hline
\end{tabular}




\section{Cureus}

\begin{tabular}{|c|c|c|c|}
\hline \multicolumn{4}{|c|}{ Ventilator use } \\
\hline Yes & 169 (81.6\%) & $38(18.4 \%)$ & $<0.001$ \\
\hline No & $126(13.9 \%)$ & $783(86.1 \%)$ & \\
\hline \multicolumn{4}{|c|}{ Intensive care unit admission } \\
\hline Yes & $195(81.9 \%)$ & $43(18.1 \%)$ & $<0.001$ \\
\hline No & $100(11.4 \%)$ & $778(88.6 \%)$ & \\
\hline \multicolumn{4}{|c|}{ Diabetes } \\
\hline Yes & $143(28.7 \%)$ & $356(71.3 \%)$ & 0.13 \\
\hline No & $152(24.6 \%)$ & $465(75.4 \%)$ & \\
\hline \multicolumn{4}{|c|}{ Hypertension } \\
\hline Yes & $197(29.2 \%)$ & 477 (70.8\%) & 0.009 \\
\hline No & $98(22.2 \%)$ & $344(77.8 \%)$ & \\
\hline \multicolumn{4}{|c|}{ Chronic kidney disease } \\
\hline Yes & $80(35.2 \%)$ & $147(64.8 \%)$ & $<0.001$ \\
\hline No & $215(24.2 \%)$ & $674(75.8 \%)$ & \\
\hline \multicolumn{4}{|c|}{ Acute kidney injury } \\
\hline Yes & $137(46.6 \%)$ & $157(53.4 \%)$ & $<0.001$ \\
\hline No & $158(19.2 \%)$ & $664(80.8 \%)$ & \\
\hline \multicolumn{4}{|c|}{ Congestive heart failure } \\
\hline Yes & $73(37.6 \%)$ & $121(62.4 \%)$ & $<0.001$ \\
\hline No & $222(24.1 \%)$ & $700(75.9 \%)$ & \\
\hline \multicolumn{4}{|c|}{ Chronic obstructive pulmonary disease } \\
\hline Yes & $56(35.7 \%)$ & $101(64.3 \%)$ & 0.005 \\
\hline No & $239(24.9 \%)$ & $720(75.1 \%)$ & \\
\hline \multicolumn{4}{|c|}{ Cerebrovascular accident (stroke) } \\
\hline Yes & $25(34.2 \%)$ & $48(65.8 \%)$ & 0.117 \\
\hline No & $270(25.9 \%)$ & $773(74.1 \%)$ & \\
\hline \multicolumn{4}{|c|}{ Coronary artery disease } \\
\hline Yes & $76(36.9 \%)$ & $130(73.6 \%)$ & $<0.001$ \\
\hline No & $219(24.1 \%)$ & 691 (75.9\%) & \\
\hline \multicolumn{4}{|c|}{ Use of ACEi or ARBs } \\
\hline Yes & $64(22.9 \%)$ & $216(77.1 \%)$ & 0.117 \\
\hline No & $231(27.6 \%)$ & $605(72.4 \%)$ & \\
\hline \multicolumn{4}{|c|}{ Use of beta-blocker } \\
\hline Yes & $108(31.2 \%)$ & $238(68.8 \%)$ & 0.015 \\
\hline No & $187(24.3 \%)$ & $583(75.7 \%)$ & \\
\hline \multicolumn{4}{|c|}{ Use of calcium channel blocker } \\
\hline Yes & $89(32.4 \%)$ & $186(67.6 \%)$ & 0.01 \\
\hline No & $206(24.5 \%)$ & 635 (75.5\%) & \\
\hline
\end{tabular}




\section{Cureus}
Use of statin
Yes
$140(28.2 \%)$
$357(71.8 \%)$
0.239
No
$155(25 \%)$
$464(75 \%)$
Use of steroid
Yes
$239(32.5 \%)$
$496(67.5 \%)$
$<0.001$
No
$56(14.7 \%)$
$325(85.3 \%)$

TABLE 1: Bivariate analysis of the relationship between categorical variables and mortality

ACEi: angiotensin-converting enzyme inhibitors; ARB: angiotensin receptor blockers

In the bivariate analysis of the continuous variables, longer duration of hospital stay $(\mathrm{p}<0.001)$, older age $(p<0.001)$, and higher admission C-reactive protein (CRP) $(p<0.001)$, lactate dehydrogenase (LDH) $(p<0.001)$, $d$-dimer $(p<0.001)$, ferritin $(p=0.04)$, troponin $(p<0.001)$, platelet count $(p<0.001)$, white blood cell count $(\mathrm{p}<0.001)$, potassium $(\mathrm{p}<0.001)$, and creatinine $(<0.001)$ were associated with increased mortality (Table 2$)$. Higher body mass index (BMI) $(\mathrm{p}=0.75)$ was not associated with increased mortality (Table 2$)$. 


\section{Cureus}

\begin{tabular}{|c|c|c|c|c|c|}
\hline & Expired & Number of patients & Mean & Standard deviation & p-Value \\
\hline \multirow{2}{*}{ Length of stay (days) } & No & 821 & 7.72 & 7.21 & $<0.001$ \\
\hline & Yes & 295 & 12.66 & 9.57 & \\
\hline \multirow{2}{*}{ Age (years) } & No & 821 & 63.48 & 18.31 & $<0.001$ \\
\hline & Yes & 295 & 71.20 & 13.53 & \\
\hline \multirow{2}{*}{ Body mass index } & No & 821 & 30.84 & 8.85 & 0.75 \\
\hline & Yes & 295 & 30.65 & 9.06 & \\
\hline \multirow{2}{*}{ C-reactive protein } & No & 636 & 8.00 & 6.22 & $<0.001$ \\
\hline & Yes & 249 & 11.63 & 6.43 & \\
\hline \multirow{2}{*}{ Lactate dehydrogenase } & No & 626 & 339.05 & 433.13 & $<0.001$ \\
\hline & Yes & 238 & 581.04 & 626.22 & \\
\hline \multirow{2}{*}{ D-dimer } & No & 691 & 1098.00 & 1319.16 & $<0.001$ \\
\hline & Yes & 257 & 1954.26 & 1612.68 & \\
\hline \multirow{2}{*}{ Ferritin } & No & 470 & 662.14 & 2430.87 & 0.04 \\
\hline & Yes & 211 & 1107.52 & 2973.51 & \\
\hline \multirow{2}{*}{ Troponin } & No & 747 & 0.13 & 0.53 & $<0.001$ \\
\hline & Yes & 277 & 0.43 & 1.69 & \\
\hline \multirow{2}{*}{ Creatine phosphokinase } & No & 557 & 254.89 & 736.69 & $<0.001$ \\
\hline & Yes & 217 & 850.33 & 3985.84 & \\
\hline \multirow{2}{*}{ Platelet } & No & 812 & 254.36 & 114.64 & $<0.001$ \\
\hline & Yes & 278 & 221.23 & 102.39 & \\
\hline \multirow{2}{*}{ White blood cell } & No & 819 & 9.10 & 5.16 & $<0.001$ \\
\hline & Yes & 281 & 12.32 & 6.65 & \\
\hline \multirow{2}{*}{ Potassium } & No & 814 & 4.18 & 0.63 & $<0.001$ \\
\hline & Yes & 280 & 4.49 & 0.82 & \\
\hline \multirow{2}{*}{ Creatinine } & No & 814 & 1.42 & 1.99 & $<0.001$ \\
\hline & Yes & 280 & 2.13 & 2.38 & \\
\hline
\end{tabular}

\section{TABLE 2: Bivariate analysis of the relationship between continuous variables and mortality}

\section{Multivariate analysis}

After adjusting for co-variables including patients' demographics, comorbidities, laboratory results on admission and other medications that patients received while hospitalized, age $(\mathrm{p}<0.001)$, admission to ICU $(p<0.001), C R P(p<0.001), L D H(p=0.039)$, and use of remdesivir $(0.018)$ were independently associated with mortality in COVID-19 patients in our study (Table 3). 


\section{Cureus}

\begin{tabular}{|c|c|c|c|c|c|c|}
\hline & \multirow{2}{*}{ Regression coefficient } & \multirow{2}{*}{ Standard error } & \multirow{2}{*}{ p-Value } & \multirow{2}{*}{ Hazard ratio } & \multicolumn{2}{|c|}{$95 \% \mathrm{Cl}$ for hazard ratio } \\
\hline & & & & & Lower & Upper \\
\hline Intensive care unit admission & -1.059 & 0.159 & 0.000 & 0.347 & 0.254 & 0.474 \\
\hline C-reactive protein & 0.042 & 0.011 & 0.000 & 1.043 & 1.021 & 1.066 \\
\hline Lactate dehydrogenase & 0.000 & 0.000 & 0.039 & 1.000 & 1.000 & 1.000 \\
\hline Remdesivir use & 0.337 & 0.143 & 0.018 & 1.401 & 1.059 & 1.853 \\
\hline Age & 0.034 & 0.006 & 0.000 & 1.034 & 1.023 & 1.046 \\
\hline
\end{tabular}

TABLE 3: Multivariate cox regression (proportional hazard) analysis of factors that affect mortality in COVID-19 patients

Cl: confidence interval; COVID-19: coronavirus disease 2019

A one-year increase in age was associated with 1.03 increased risk of mortality. Admission to the ICU resulted in 0.35 decreased risk of mortality. A one-unit increase in CRP was associated with 1.04 increased risk of mortality and one-unit increase in LDH is associated with 1.0 increased risk of mortality.

Gender, race, BMI, presence of co-morbidities such as diabetes and hypertension, and use of steroids, betablockers, ACEi, or ARBs were not associated with mortality in the multivariate analysis (Table 3).

\section{Discussion}

Our analysis of mortality predictors in COVID-19 patients admitted in two hospitals in Southern California found multiple independent predictors of mortality. While some of our findings were consistent with findings in previous studies, other findings were unexpected. Consistent with prior studies, increased age appears to be a strong risk factor for COVID-19 mortality in our study. A one-year increase in age was associated with 1.03 increased risk of mortality after adjusting for other co-variables. Age has consistently been found to be an important independent risk factor for both severe disease and mortality in COVID-19 patients and mortality increases with age, with the highest mortality reported among people over 80 years of age $[2,9-12]$.

Our study found that being admitted in ICU resulted in 0.35 decreased risk of mortality. In our study, $82 \%$ of patients who were admitted to the ICU died and we had expected that ICU admission had no effect on mortality because of the high mortality rate of those admitted to ICU. However, contrary to our expectation, being admitted to the ICU significantly increased the chances of survival. In some health facilities, including the facilities in our study, due to pandemic pressures on limited ICU services, there was widespread use of advanced respiratory support (non-invasive ventilation or high-flow nasal oxygen) outside ICUs and ICU admission was reserved for patients that were deemed to be more severe [13]. However, based on the result of this study, severe or critically ill COVID-19 patients who were not admitted to the ICU, including patients who requested not to be resuscitated or intubated, were more likely to die compared to those that were admitted to ICU. Patients who requested not to be resuscitated or intubated were usually not admitted to the ICU irrespective of their sickness severity and this might explain the mortality benefit with ICU admission. Unfortunately, we did not have data on the number of COVID-19 patients who requested not to be resuscitated or intubated and could not adjust for these in our regression model. Conversely, close monitoring and early intervention for patients in ICU could explain the mortality benefit seen with ICU admission. However, our study did not show any mortality benefit with ventilator use.

Furthermore, we found that the use of remdesivir was associated with increased risk of mortality after adjusting for patients' demographics, comorbidities, laboratory results on admission, and other medications that patients received while hospitalized. Though remdesivir has been found to be beneficial when used early in the course of COVID-19 illness, studies have shown that, similar to our finding, it may not be beneficial in the later stage of COVID-19 illness or in critically ill patients requiring mechanical ventilation [14-17]. This is because while remdesivir may be beneficial in the initial phase of viral replication in COVID19 patients, severe disease in Covid-19 patient is mainly caused by the host's aggressive inflammatory response against COVID-19 virus and remdesivir might not be very beneficial at that stage $[18,19]$. The reason for the increased mortality with remdesivir in our study is not clear but one possible explanation is that the increased mortality could be secondary to remdesivir-associated bradycardia. The use of remdesivir has been associated with bradycardia in COVID-19 patients and bradycardia has been associated with increased mortality in COVID-19 patients [20-22]. 
The use of corticosteroids including dexamethasone or methylprednisolone was not associated with decreased mortality in our study after adjusting for patients' demographics, comorbidities, laboratory results on admission, and other medications that patients received while hospitalized. Prior studies have shown that use of steroids lowers mortality among severe or critically ill patients who were and were not receiving invasive mechanical ventilation, irrespective of patients' age or gender by modulating inflammation-mediated lung injury and thereby reducing progression to respiratory failure and death $[23,24]$. Both high- and low-dose steroid use has been associated with decreased mortality, and, currently, there was no evidence suggesting that a higher dose of steroid is associated with significant greater benefit than a lower dose $[23,25]$. In the landmark RECOVERY trial that showed that dexamethasone has mortality benefit in COVID- 19 patients, less than $0.1 \%$ of patients received concomitant remdesivir and the study did not report the proportions of patients who were on high-flow device or non-invasive ventilation or the amount of oxygen that patients received [24]. In our study, $60 \%$ of the patients who received steroid also received remdesivir and the lack of mortality benefit with steroid seen in our study may be due to the difference between our study population and the studies that showed mortality benefit with steroids.

We found that high CRP and LDH levels were associated with increased risk of mortality. A one-unit increase in CRP was associated with 1.04 increased risk of mortality and one-unit increase in LDH was associated with 1.0 increased risk of mortality. LDH and CRP are markers of acute inflammation and hypoxia in COVID-19 patients and increased levels of CRP and LDH correlate with increased severity of illness and increased risk of death [26-28].

Our study has some limitations. First, our study is a retrospective observational study and though we adjusted for major comorbidities and confounders in our analysis (Tables 1,2), we may not have adjusted for some unmeasured or unknown confounders. However, we did not have data on the number of COVID-19 patients who requested not to be resuscitated or intubated and those who received COVID-19 vaccine and could not adjust for these in our regression model. Secondly, patients' medications included in the analysis were based on information in the hospital electronic medical record which may not capture all the medication patients were taking outpatient and could have led to misclassification bias; however, we believe that the chance of this happening is minimal and will not have substantially affected the result of our study. Thirdly, the findings of our study are only generalizable to hospitalized COVID-19 population.

\section{Conclusions}

In conclusion, this is a retrospective study of 1,116 COVID-19 patients who were admitted at two hospitals in Southern California, United States, between March 2020 and March 2021. After adjusting for independent variables, older age, high levels of CRP and LDH, and use of remdesivir were associated with increased mortality in our study. Conversely, admission to ICU was associated with decreased mortality while there was no mortality benefit with the use of steroid, statin, calcium channel blockers, or ACEi and ARB. Increased BMI, hypertension, and diabetes were not independently associated with mortality. There is need for more research on the mortality benefit of ICU admission for COVID-19 patients who request not to be resuscitated or intubated. There is also need for more studies on the mortality benefit of concurrent use of remdesivir and steroids in COVID-19 patients.

\section{Additional Information Disclosures}

Human subjects: Consent was obtained or waived by all participants in this study. WIRB-Copernicus Group (WCG) IRB issued approval 13410516. Animal subjects: All authors have confirmed that this study did not involve animal subjects or tissue. Conflicts of interest: In compliance with the ICMJE uniform disclosure form, all authors declare the following: Payment/services info: All authors have declared that no financial support was received from any organization for the submitted work. Financial relationships: All authors have declared that they have no financial relationships at present or within the previous three years with any organizations that might have an interest in the submitted work. Other relationships: All authors have declared that there are no other relationships or activities that could appear to have influenced the submitted work.

\section{References}

1. Parasher A: COVID-19: current understanding of its pathophysiology, clinical presentation and treatment . Postgrad Med J. 2021, 97:312-20. 10.1136/postgradmedj-2020-138577

2. World Health Organization. Report of the WHO-China Joint Mission on Coronavirus Disease 2019 (COVID19). (2020). Accessed: June 10, 2021: https://www.who.int/docs/default-source/coronaviruse/who-chinajoint-mission-on-covid-19-final-report.pdf.

3. CDC. First Travel-related Case of 2019 Novel Coronavirus Detected in United States . (2020). Accessed: June 10, 2021: https://www.cdc.gov/media/releases/2020/p0121-novel-coronavirus-travel-case.html..

4. CDC. United States COVID-19 Cases, Deaths, and Laboratory Testing (NAATs) by State, Territory, and Jurisdiction. (2021). Accessed: June 10, 2021: https://covid.cdc.gov/covid-data-tracker/.

5. Tian W, Jiang W, Yao J, et al.: Predictors of mortality in hospitalized COVID-19 patients: a systematic review and meta-analysis. J Med Virol. 2020, 92:1875-83. 10.1002/jmv.26050

6. Mesas AE, Cavero-Redondo I, Álvarez-Bueno C, Sarriá Cabrera MA, Maffei de Andrade S, Sequí-Dominguez 
I, Martínez-Vizcaíno V: Predictors of in-hospital COVID-19 mortality: a comprehensive systematic review and meta-analysis exploring differences by age, sex and health conditions. PLoS One. 2020, 15:e0241742. 10.1371/journal.pone.0241742

7. Imam Z, Odish F, Gill I, et al.: Older age and comorbidity are independent mortality predictors in a large cohort of 1305 COVID-19 patients in Michigan, United States. J Intern Med. 2020, 288:469-76. 10.1111/joim.13119

8. Aly MH, Rahman SS, Ahmed WA, Alghamedi MH, Al Shehri AA, Alkalkami AM, Hassan MH: Indicators of critical illness and predictors of mortality in COVID-19 patients. Infect Drug Resist. 2020, 13:1995-2000. 10.2147/IDR.S261159

9. Romero Starke K, Petereit-Haack G, Schubert M, Kämpf D, Schliebner A, Hegewald J, Seidler A: The agerelated risk of severe outcomes due to COVID-19 infection: a rapid review, meta-analysis, and metaregression. Int J Environ Res Public Health. 2020, 17:5974. 10.3390/ijerph17165974

10. Chen T, Dai Z, Mo P, et al.: Clinical characteristics and outcomes of older patients with coronavirus disease 2019 (COVID-19) in Wuhan, China: a single-centered, retrospective study. J Gerontol A Biol Sci Med Sci. 2020, 75:1788-95. 10.1093/gerona/glaa089

11. Posso M, Comas M, Román M, et al.: Comorbidities and mortality in patients with COVID-19 aged 60 years and older in a university hospital in Spain. Arch Bronconeumol (Engl Ed). 2020, 56:756-8. 10.1016/j.arbres.2020.06.012

12. O'Driscoll M, Ribeiro Dos Santos G, Wang L, et al.: Age-specific mortality and immunity patterns of SARSCoV-2. Nature. 2021, 590:140-5. 10.1038/s41586-020-2918-0

13. Armstrong RA, Kane AD, Cook TM: Outcomes from intensive care in patients with COVID-19: a systematic review and meta-analysis of observational studies. Anaesthesia. 2020, 75:1340-9. 10.1111/anae.15201

14. Young B, Tan TT, Leo YS: The place for remdesivir in COVID-19 treatment . Lancet Infect Dis. 2021, 21:201. 10.1016/S1473-3099(20)30911-7

15. Beigel JH, Tomashek KM, Dodd LE, et al.: Remdesivir for the treatment of Covid-19 - final report . N Engl J Med. 2020, 383:1813-26. 10.1056/NEJMoa2007764

16. Garibaldi BT, Wang K, Robinson ML, et al.: Comparison of time to clinical improvement with vs without remdesivir treatment in hospitalized patients with COVID-19. JAMA Netw Open. 2021, 4:e213071. 10.1001/jamanetworkopen.2021.3071

17. Wang Y, Zhang D, Du G, et al.: Remdesivir in adults with severe COVID-19: a randomised, double-blind, placebo-controlled, multicentre trial. Lancet. 2020, 395:1569-78. 10.1016/S0140-6736(20)31022-9

18. Tay MZ, Poh CM, Rénia L, MacAry PA, Ng LF: The trinity of COVID-19: immunity, inflammation and intervention. Nat Rev Immunol. 2020, 20:363-74. 10.1038/s41577-020-0311-8

19. Ruan Q, Yang K, Wang W, Jiang L, Song J: Clinical predictors of mortality due to COVID-19 based on an analysis of data of 150 patients from Wuhan, China. Intensive Care Med. 2020, 46:846-8. 10.1007/s00134020-05991-x

20. Gubitosa JC, Kakar P, Gerula C, et al.: Marked sinus bradycardia associated with remdesivir in COVID-19: a case and literature review. JACC Case Rep. 2020, 2:2260-4. 10.1016/j.jaccas.2020.08.025

21. Touafchia A, Bagheri H, Carrié D, Durrieu G, Sommet A, Chouchana L, Montastruc F: Serious bradycardia and remdesivir for coronavirus 2019 (COVID-19): a new safety concerns. Clin Microbiol Infect. 2021, 27:791.e5-791.e8. 10.1016/j.cmi.2021.02.013

22. Kumar S, Arcuri C, Chaudhuri S, Gupta R, Aseri M, Barve P, Shah S: A novel study on SARS-COV-2 virus associated bradycardia as a predictor of mortality-retrospective multicenter analysis. Clin Cardiol. 2021, 44:857-62. 10.1002/clc.23622

23. Sterne JA, Murthy S, Diaz JV, et al.: Association between administration of systemic corticosteroids and mortality among critically ill patients with COVID-19: a meta-analysis. JAMA. 2020, 324:1330-41. 10.1001/jama.2020.17023

24. Horby P, Lim WS, Emberson JR, et al.: Dexamethasone in hospitalized patients with Covid-19. N Engl J Med. 2021, 384:693-704. 10.1056/NEJMoa2021436

25. Piccica M, Lagi F, Trotta M, Spinicci M, Zammarchi L, Bartoloni A; COCORA Working Group: High-dose steroids for the treatment of severe COVID-19. Intern Emerg Med. 2021, 16:1395-9. 10.1007/s11739-02102707-x

26. Li C, Ye J, Chen Q, et al.: Elevated lactate dehydrogenase (LDH) level as an independent risk factor for the severity and mortality of COVID-19. Aging (Albany NY). 2020, 12:15670-81. 10.18632/aging.103770

27. Aloisio E, Chibireva M, Serafini L, Pasqualetti S, Falvella FS, Dolci A, Panteghini M: A comprehensive appraisal of laboratory biochemistry tests as major predictors of COVID-19 severity. Arch Pathol Lab Med. 2020, 144:1457-64. 10.5858/arpa.2020-0389-SA

28. Huang I, Pranata R, Lim MA, Oehadian A, Alisjahbana B: C-reactive protein, procalcitonin, D-dimer, and ferritin in severe coronavirus disease-2019: a meta-analysis. Ther Adv Respir Dis. 2020, 14:1753466620937175. 10.1177/1753466620937175 